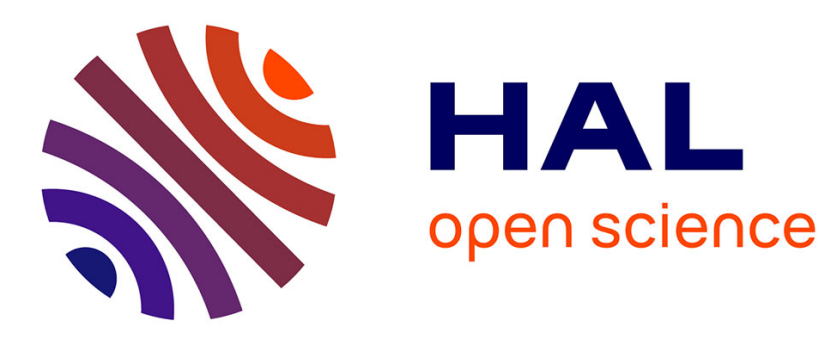

\title{
Efficient System Capacity User Selection Algorithm in MU-MIMO
}

Malo Manini, Cédric Gueguen, Rodolphe Legouable, Xavier Lagrange

\section{To cite this version:}

Malo Manini, Cédric Gueguen, Rodolphe Legouable, Xavier Lagrange. Efficient System Capacity User Selection Algorithm in MU-MIMO. VTC2021-Spring: IEEE 93rd Vehicular Technology Conference, Apr 2021, Helsinki, Finland. 10.1109/VTC2021-Spring51267.2021.9448632 . hal-03265005

\section{HAL Id: hal-03265005 https://hal.science/hal-03265005}

Submitted on 18 Jun 2021

HAL is a multi-disciplinary open access archive for the deposit and dissemination of scientific research documents, whether they are published or not. The documents may come from teaching and research institutions in France or abroad, or from public or private research centers.
L'archive ouverte pluridisciplinaire $\mathbf{H A L}$, est destinée au dépôt et à la diffusion de documents scientifiques de niveau recherche, publiés ou non, émanant des établissements d'enseignement et de recherche français ou étrangers, des laboratoires publics ou privés. 


\title{
Efficient System Capacity User Selection Algorithm in MU-MIMO
}

\author{
Malo Manini, Cédric Gueguen, Rodolphe Legouable, Xavier Lagrange \\ b-com, University of Rennes1/IRISA, IMT Atlantique/IRISA \\ France \\ Email: \{malo.manini, rodolphe.legouable,\}@b-com.com; \{cedric.gueguen\}@irisa.fr; \{xavier.lagrange\}@imt-atlantique.fr;
}

\begin{abstract}
Radio resource allocation is a major research field in wireless networks. The main challenge is to find the most suitable user for each time and frequency resource to ensure the Quality of Service $(\mathrm{QoS})$ requirements. The use of Massive Multiple-Input-Multiple-Output (MIMO) makes it possible to simultaneously schedule several users on the same time and frequency resources. This is a new paradigm for resource scheduling. In this context, resource allocation algorithms have to account for the choice of users served on the same time and frequency resource in their decision mechanism. In this paper we analyze the impact of different group sizes on system capacity using several user selection algorithms and we propose a new user selection algorithm which performs closer to the optimal algorithm proposed in the literature.

Index Terms-5G, Massive-MU-MIMO, User selection, Resource allocation.
\end{abstract}

\section{INTRODUCTION}

One of the key technologies for $5 \mathrm{G}$ radio transmission is Massive-MIMO [1]. Having a larger number of antennas at the base station can significantly improve the link budget by focusing the energy in the chosen direction [2]. Focusing the energy can also be used to serve multiple users (MU) on the same time and frequency resource. This technology opens the way to conceiving new scheduling algorithms in order to make the most of Massive-MIMO.

In MU mode, when several users are served on the same time-frequency unit, they are said to be in the same group. The total system rate is expected to be higher compare to single user (SU) mode. However, there is intracell interference, which increases with the number of users by group and reduces the bit rate. Reducing interference is crucial but done at the expense of individual throughput. Indeed, an efficient grouping strategy is necessary to find the best compromise between interference reduction and individual throughput.

For such strategies to be developed, efficient indicators are required. Papers from the literature suggest using the correlation of users' channel matrices [3], [4] as grouping indicator. In our previous study [5] we noticed that this correlation was not a suitable indicator for user selection in our simulating context, due to its instability and lack of accuracy in predicting the throughput impact.

The objective of this paper is to propose a new indicator for group selection in a MU-MIMO context. First, we study different user selection algorithms and propose a new solution based on previous user selections. Then, we evaluate the impact of group size on overall system throughput. Our solution, called Efficient System Capacity User Selection (ESCUS), progressively builds its indicator upon previous users' selections by storing users' throughput and selecting groups of pairs of users. As the system's capacity to manage more users simultaneously increases, so does the algorithm's complexity. This indicator depends on the obtained throughput between pairs of users and does not increase in complexity when the number of users per group increases. Thanks to channel stability due to channel hardening [6], this indicator is stable in time and frequency. Results show a strong increase in the system throughput compared to a correlation based algorithm.

The paper is organized as follows. Section II provides a detailed description of the system in question. Section III presents our new algorithm proposal for user selection. Section IV introduces the main parameters of our simulation setup and the performance evaluation of our new solution. Section V concludes the paper.

\section{DESCRIPTION OF THE TRANSMISSION CHAIN}

We consider one base station (BS, also called gNB) and several pieces of user equipment (UE) and study the downlink transmission. In order to maintain our simulation time a reasonable limit, each simulation frame has a duration of $10 \mathrm{~ms}$ with a usable bandwidth of $2 \mathrm{MHz}$. This frame is divided into resource units (RU) with $60 \mathrm{kHz}$ bandwidth and $1 \mathrm{~ms}$ length. We define $B$ as the bandwidth of the RU, $\mathcal{U}$ as the set of all UEs in the cell, $\mathcal{A}$ as the set of all UE allocated simultaneously to the same RU, and $n_{\mathrm{t}}, n_{\mathrm{r}}$ the number of antennas at the transmitter (BS) and at the receiver (UE), respectively. The channel between the set of antennas at the BS and the set of antennas at UE $i$ is modelled by complex matrix $\mathbf{H}_{i}$. The precoding matrix at the BS is represented by matrix $\mathbf{F}_{i}$ and the digital combining matrix at UE $i$ is represented by $\mathbf{W}_{i}^{*}$. The white noise average power is denoted by $\sigma_{\mathrm{N}}^{2}$. Note that interference from neighbouring base stations can be integrated in $\sigma_{\mathrm{N}}^{2}$.

The channel matrix of a UE $i$ is given by $\mathbf{H}_{i}[t] \in \mathbb{C}^{n_{\mathrm{r}} \times n_{\mathrm{t}}}$ and is perfectly known at the gNB. Matrix $\mathbf{H}_{i}$ is generated using the Saleh-Valenzuela model [16] extended to millimeter waves. The advantage of this channel model is that it can be validated with measured data. The total number of parallel transmissions that can be made on the same RU by one gNB is given by $n_{\mathrm{t}}$. The maximum number of transmissions on the 
same $\mathrm{RU}$ to a given $\mathrm{UE}$ is $\min \left(n_{\mathrm{t}}, n_{\mathrm{r}}\right)$. The time is considered discrete and $\Delta t$ is equivalent to a timeslot. We assume that the coherence time of the channel is greater than $\Delta t$ and therefore greater than the scheduling time.

\section{A. Transmission model}

At a given time, scheduling can be viewed as an indicator function: $\delta_{i}(t)$ where $i$ is the UE index and $\delta_{i}(t) \in$ $\left[0, \min \left(n_{\mathrm{t}}, n_{\mathrm{r}}\right)\right]$ gives the number of streams on the resource allocated to $i$.

We have the following constraint:

$$
\sum_{i \in \mathcal{U}} \delta_{i}(t) \leq n_{\mathrm{t}}
$$

The potential number of usable resources is given by $n_{\mathrm{t}} \times K$, where $K$ is the number of resource units. We assume that there is no limitation regarding the number of RF chains and the processing capacity. To illustrate the previous equation, we can consider a number of UEs equal to $n_{\mathrm{t}} \times K$, for example $n_{\mathrm{t}} \times K=100$. In this case, UEs will only have $1 \mathrm{RU}$ among 100 on average per timeslot.

For the sake of clarity we omit $t$ for this part. We denote the precoding matrix associated with channel $\mathbf{H}_{i}$ as $\mathbf{F}_{i} \in \mathbb{C}^{n_{\mathrm{t}} \times \delta_{i, k}}$. The dimension of $\mathbf{F}_{i}$ depends on the number of antennas at the gNB and the selected streams. The signal transmitted to $\mathrm{UE} i$ is :

$$
\mathbf{x}_{i}=\mathbf{F}_{i} \mathbf{s}_{i}
$$

where $i \in \mathcal{U}$ and $\mathbf{s}_{i}$ is the $\delta_{i} \times 1$ transmitted vector of symbols at a given subcarrier. Note that $\mathbf{s}_{i}$ is null if $\delta_{i}=0$. The overall signal transmitted by the base station is:

$$
\mathbf{X}=\sum_{j \in \mathcal{U}, \delta_{j}>0} \mathbf{F}_{j} \mathbf{s}_{j} .
$$

At the receiver, the signal is affected by the channel matrix $\mathbf{H}_{i}$, noise corrupting the received signal $\mathbf{n}$ of power $\sigma^{2}$ and $\mathbf{I}$ external cell interference:

$$
\mathbf{y}_{i}=\underbrace{\mathbf{H}_{i} \mathbf{X}}_{\text {signal }}+\underbrace{\mathbf{I}+\mathbf{n}}_{\text {external interference }+ \text { noise }} .
$$

Due to the fact that we are considering a Multi-User transmission (MU-MIMO) the transmitted signal for other UEs scheduled on the same RU has to be considered as internal interference:

$$
\mathbf{y}_{i}=\underbrace{\mathbf{H}_{i} \mathbf{F}_{i} \mathbf{s}_{i}}_{\text {signal }}+\underbrace{\sum_{j \neq i, \delta_{j}>0} \mathbf{H}_{i} \mathbf{F}_{j} \mathbf{s}_{j}+(\mathbf{I}+\mathbf{n})}_{\text {interference }+ \text { noise }} .
$$

This internal interference depends on the precoders' $\mathbf{F}_{j}$ orthogonality to the channel matrix $\mathbf{H}_{i}$. The signal at the receiver after combining is finally given by:

$$
\mathbf{z}_{i}=\mathbf{W}_{i}^{*} \mathbf{H}_{i} \mathbf{F}_{i} \mathbf{s}_{i}+\mathbf{W}_{i}^{*} \sum_{j \neq i, \delta_{j}>0} \mathbf{H}_{i} \mathbf{F}_{j} \mathbf{s}_{j}+\mathbf{W}_{i}^{*}(\mathbf{n}+\mathbf{I})
$$

where $\mathbf{W}_{i}^{*}[k]$ is the digital combining matrix [7] and $(.)^{*}$ the conjugate transpose of a complex matrix.

\section{B. Precoding techniques}

We consider two precoding methods:

1) singular-value decomposition (SVD): A MIMO channel $\mathbf{H}_{i}$ of a user $i$ can be decomposed using the SVD as follow:

$$
\mathbf{H}_{i}=U_{i} \Sigma_{i} V_{i}^{*} .
$$

The optimal precoder in SU-MIMO for $i$ is $\mathbf{F}_{i}$ [2]:

$$
\mathbf{F}_{i}=V_{i} \Lambda^{1 / 2},
$$

where $\Lambda^{1 / 2}$ is a diagonal matrix of which the element $\lambda_{i}$ scales the power transmitted.

2) Block Diagonal precoding [8][9]: In MU-MIMO several users can be scheduled simultaneously on the same RU. In such conditions, users can experience interference from other users. The objective of the Block Diagonal precoder is to eliminate UEs interference when they are scheduled in a MU-MIMO context. Block Diagonal is similar to SVD, with the addition of a prior step of interference cancelling. Therefore, all users that are simultaneously scheduled have to be considered in the precoding process.

We define $\tilde{\mathbf{H}}_{i}^{\mathrm{T}}$ the concatenation of channel matrices of all users in $\mathcal{A}$ except $i$ :

$$
\tilde{\mathbf{H}}_{i}^{\mathrm{T}}=\left[\mathbf{H}_{1}^{*} \ldots \mathbf{H}_{i-1}^{*} \mathbf{H}_{i+1}^{*} \ldots \mathbf{H}_{A}^{*}\right]^{*},
$$

where $A=\operatorname{card}(\mathcal{A})$ is the number of elements in set $\mathcal{A}$. Note that $\tilde{\mathbf{H}}_{i}^{\mathrm{T}}$ is a $n_{\mathrm{r}} \times(A-1)$ rows and $n_{\mathrm{t}}$ columns complex matrix. We are using the SVD on $\tilde{\mathbf{H}}_{i}^{\mathrm{T}}$.

$$
\tilde{\mathbf{H}}_{i}^{\mathrm{T}}=U_{i}^{\mathrm{T}} \Sigma_{i}\left[V_{i}^{(1)} V_{i}^{(0)}\right]^{*},
$$

where $\left[V_{i}^{(1)} V_{i}^{(0)}\right]$ is a $n_{\mathrm{t}} \times n_{\mathrm{t}}$ matrix. $V_{i}^{(1)}$ contains vectors corresponding to nonzero singular values and $V_{i}^{(0)}$ contains vectors corresponding to zero singular values.

$$
\tilde{\mathbf{H}}_{i}^{\mathrm{T}} \tilde{V}_{i}^{(0)}=\tilde{U}_{i} \tilde{\Sigma}_{i}\left[\tilde{V}_{i}^{(1)} \tilde{V}_{i}^{(0)}\right]^{*} .
$$

The total precoding matrix is given by:

$$
T^{B D}=\left[\tilde{V}_{1}^{(0)} V_{1}^{(1)} \tilde{V}_{2}^{(0)} V_{2}^{(1)} \ldots \tilde{V}_{A}^{(0)} V_{A}^{(1)}\right] \Lambda^{1 / 2} .
$$

\section{User Equipment throughput in MU mode}

For the MU mode, we consider the Block Diagonal (BD) precoding technique [9] because the $\mathrm{BD}$ precoder is focused on interference management and therefore limits the reduction of the bit rate due to the intracell interference of the group. The bit rate for UE $i$ is given by [10] :

$$
R_{i}=B \log _{2}\left|\mathbb{1}_{n_{\mathrm{r}}}+\frac{\mathbf{W}_{i}^{*} \mathbf{H}_{i} \mathbf{F}_{i}\left(\mathbf{W}_{i}^{*} \mathbf{H}_{i} \mathbf{F}_{i}\right)^{*}}{\mathbf{W}_{i}^{*}\left(\sigma^{2} \mathbb{1}_{n_{\mathrm{r}}}+\mathbf{G}_{i}\right) \mathbf{W}_{i}}\right|,
$$

where $\mathbb{1}_{n_{\mathrm{r}}}$ is an identity matrix of size $n_{\mathrm{r}}$ and $\mathbf{G}_{i}$ is the intracell interference of the group, which is given by:

$$
\mathbf{G}_{i}=\sum_{j \neq i, \delta_{j}>0} \mathbf{H}_{i} \mathbf{F}_{j}\left(\mathbf{H}_{i} \mathbf{F}_{j}\right)^{*} .
$$


In MU, $n_{\mathrm{t}} / n_{\mathrm{r}}$ is the limit on users served at the same time. We define $n_{s}$ as the number of simultaneously scheduled users, where $n_{s} \leq n_{\mathrm{t}} / n_{\mathrm{r}}$. The mean system rate $R$ is:

$$
R=E\left[\sum_{i=1}^{n_{s}} R_{i}\right]
$$

where $E$ is the mathematical expectation. We consider a large set of random terminals, which are deployed randomly thanks to the properties of the channel model. Thus, $\mathbf{H}_{i}$ is a random matrix. In the following, for different configurations, we study $R$, which is our main performance indicator.

\section{User Equipment throughput in $S U$ mode}

In the SU mode, $\delta_{i}$ is non-zero for only one value of $i$. Thus, there is no intracell interference and the rate $C_{i}$ is maximized. Equation (13) is simplified as:

$$
C_{i}=B \log _{2}\left|\mathbb{1}_{n_{\mathrm{r}}}+\frac{\mathbf{W}_{i}^{*} \mathbf{H}_{i} \mathbf{F}_{i}\left(\mathbf{W}_{i}^{*} \mathbf{H}_{i} \mathbf{F}_{i}\right)^{*}}{\mathbf{W}_{i}^{*}\left(\sigma^{2} \mathbb{1}_{n_{\mathrm{r}}}\right) \mathbf{W}_{i}}\right| .
$$

User throughput in SU mode is used as an indicator for classical opportunistic resource allocation schedulers. It is the main indicator for opportunistic scheduler, such as MaxSNR [11].

\section{E. Channel matrix correlation as interference management}

In MU-MIMO good interference management is crucial to fully benefit from the technique. As seen in [3] and [4] channels matrix correlation is commonly used as an indicator of the interference level between UEs. The correlation of two UEs channel matrices indicates the interference level if they are using the same RU simultaneously. The correlation of two UEs is defined by [12]:

$$
\xi(i, j)=\frac{\left|\operatorname{tr}\left(\mathbf{H}_{i} \mathbf{H}_{j}^{*}\right)\right|}{\left\|\mathbf{H}_{i}\right\|_{F}\left\|\mathbf{H}_{j}\right\|_{F}},
$$

where $(.)^{*}$ denotes the conjugate transpose operation and $\|.\| \|_{F}$ the Forbenius norm of a matrix.

One previous work [5] shows that the correlation is not astable and accurate enough indicator to be used reliably in the user selection process. This work is why we aim to find better and more robust indicators for the user selection process.

\section{NEW ALGORITHM PROPOSAL FOR USER SELECTION}

We propose a solution, called Efficient System Capacity User Selection (ESCUS), based on previous group allocations. Our solution is based on a matrix $\mathbf{M}$ that stores the capacity values obtained when 2 users are served in the same group. This matrix is gradually built from previous allocations. ESCUS relies on a $\operatorname{card}(\mathcal{U}) \times \operatorname{card}(\mathcal{U})$ matrix which is updated at the end of the allocation process.

A common strategy for user grouping is to consider user channel correlation as an indicator, as seen in [3], [4], [13]. This technique involves calculating of channel matrix correlation, which must be regularly updated and which yields mixed results. Our solution, ESCUS, has the advantage of being less complex, even when we increase the number of users by group.
This solution is highly flexible and will be easy to adapt to any MIMO system thanks to channel hardening [6].

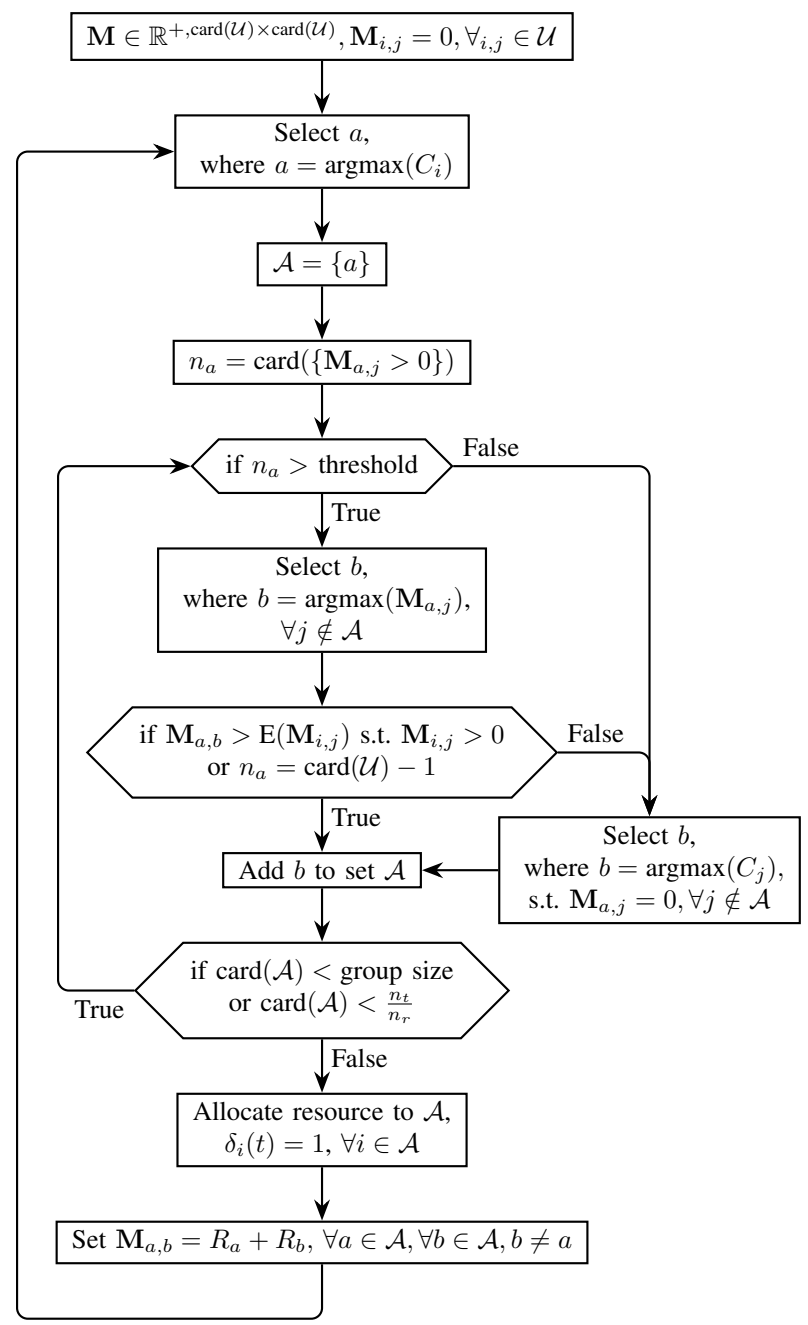

Fig. 1: Diagram of the proposed solution

ESCUS is divided into two main phases, exploration and exploitation. When the system starts, user compatibility (group quality) is unknown. During the exploration phase, ESCUS associates users in a certain order, in order to populate its database with throughput of different pairs of users. When database completion reaches a certain level, ESCUS enters an exploitation phase. During this phase, ESCUS selects users to be served depending on the best obtained throughput in the database. In each case, the database is always updated if the obtained throughput evolves. In this way, users can be served with the highest compatibility, based on their previous association.

The indicator used by ESCUS is the cumulated throughput of all pairs of users in previous groups. When the number of users per group increases, the number of possible combination increases as well. Storing the obtained throughput for each combinations would require a large database, which would grow with the number of users per group (a $n$-dimensional matrix, with $n$ the number of users per group). The larger the 
database, the longer it takes to fill it, leading to more exploration than exploitation. To avoid such constraints, the ESCUS database only stores the throughput obtained by a pair of users. The data to be stored corresponds to a $\operatorname{card}(\mathcal{U}) \times \operatorname{card}(\mathcal{U})$ matrix. Another advantage of using a bi-dimensional matrix is its flexibility concerning the number of users per group, allowing the same database structure to be used regardless of the group size. The following example illustrates the database structure. We consider a group of 3 users $\mathcal{A}=\{a, b, c\}$. ESCUS stores the sum of the rate for each possible pair set in $\mathcal{A}$. For example, $\mathbf{M}_{a, b}=\mathbf{M}_{b, a}=R_{a}+R_{b}$.

Independently from the current phase of ESCUS, the first user of the group is always selected according to a MaxSNR strategy [11] in order to maximize the total system rate. The objective of ESCUS is only to maximize the system rate. If fairness is considered, then another first-user selection strategy can be applied, such as proportional fair [14], [15].

The first phase aims at discovering unknown shared throughput between users. This exploration phase selects secondary users that have the best instantaneous SU throughput $\left(\mathcal{C}_{i}\right)$. As stated previously, this MaxSNR strategy might be replaced by another strategy to reach different goals. During this selection, only users with unknown throughput are selected when scheduled with the first user. This process stops when the number of selected users reaches the maximum group size.

When the database contains enough information, ESCUS switches to exploitation mode. The threshold for this transition depends on the system configuration and is set to $\frac{1}{4} \times \operatorname{card}(\mathcal{U})$ in our examples, as it was found to be efficient in all antenna configurations and allows for sufficient group diversity. A more configuration-specific threshold might increase performance even more.

During this second phase, ESCUS uses the data gathered previously. Secondary users are selected according to the best value in the database when associated with the first user. To prevent allocating low value, the selected value is compared to the mean of the database. When the value is lower, ESCUS switches to exploration in order to find a more suitable pair of users.

At the end of this selection process, the resource is allocated to selected users. After this allocation, the matrix $\mathbf{M}$ is updated with experienced throughput from each pair of users. This full process is described in Figure 1.

\section{Performance evaluation}

\section{A. Simulation set up}

Twenty pieces of user equipment (UEs), randomly distributed in the cell, are considered. The UE channels are mutually independent. A channel for a user $i$ is generated using the Saleh-Valenzuela model [16] extended to millimeter waves, where the obstructed-line-of-sight (OLOS) parameters are given in table I.

\begin{tabular}{|c|c|c|c|}
\hline$f$ & 26 & $\mathrm{GHz}$ & Frequency \\
\hline$\Lambda$ & 5 & $\mathrm{~ns}$ & Cluster arrival rate \\
\hline$\lambda$ & 1 & $\mathrm{~ns}$ & Ray arrival rate \\
\hline$\Gamma$ & 8.7 & $\mathrm{~ns}$ & Cluster decay rate \\
\hline$\gamma$ & 4.7 & $\mathrm{~ns}$ & Ray decay rate \\
\hline$\sigma$ & 0.1 & $\mathrm{rad}$ & Intra-cluster angles standard deviation \\
\hline
\end{tabular}

TABLE I: Channel model parameters, from [16]

We consider one sector in a typical 3-sector configuration: the angle between the terminal and the base station is between $0^{\circ}$ and $120^{\circ}$ horizontally and between $-45^{\circ}$ and $45^{\circ}$ vertically. Antenna arrays at the base station and on the user side are Uniform Linear Array (ULA), with $\frac{\lambda}{2}$ distance between antenna elements, where $\lambda$ is the wavelength of the central frequency. We assume that the base station has full knowledge of the channel for each UE.

ESCUS is compared to several simple and known algorithms:

- 1stMaxSNR: a MaxSNR for the first user and then all secondary users of the group are chosen using a random function.

- FullMaxSNR: a MaxSNR for the first user and then all secondary users of the group are chosen depending on their SNR.

- Correlation-Based-User-Selection (CBUS) [3]: a MaxSNR for the first user and then all secondary users in the group are chosen according to the lowest correlation with the first user.

- ESCUS: our proposed solution, which is described in Section III

- Optimal: the exhaustive search for the best group. This optimal solution guarantees the maximum system throughput.

As a representative correlation based algorithm, CBUS presents the advantage of focusing only on system rate maximization, as opposed to fairness-oriented algorithm [4] which are not directly comparable to our solution. Two types of performance evaluation were conducted, either with a small system or with larger systems (with 20 and 60 users in the system, respectively). A first study with a small system allowed us to compare our solution with all of the aforementioned strategies, including the optimal one. A second study focused on Massive-MIMO systems, where large groups of users can be simultaneously allocated. However, maximizing the number of users per group did not always provide the best system capacity, due to a strong increase in inter-group interference. Another limiting factor is the number of RF chains, which defines the limit of group size. A 3GPP technical report [17] sets the maximum number of RF chains at 12 . At this group size, it is not possible to compute an optimal solution in reasonable time, due to its polynomial complexity depending on the number of users per group. Indeed, the throughputs of all possible groups are computed and compared. The optimal strategy is then excluded in section IV-C. Results are obtained with the mean of 100 frame samples, to avoid a nonrepresentative statistical event. 


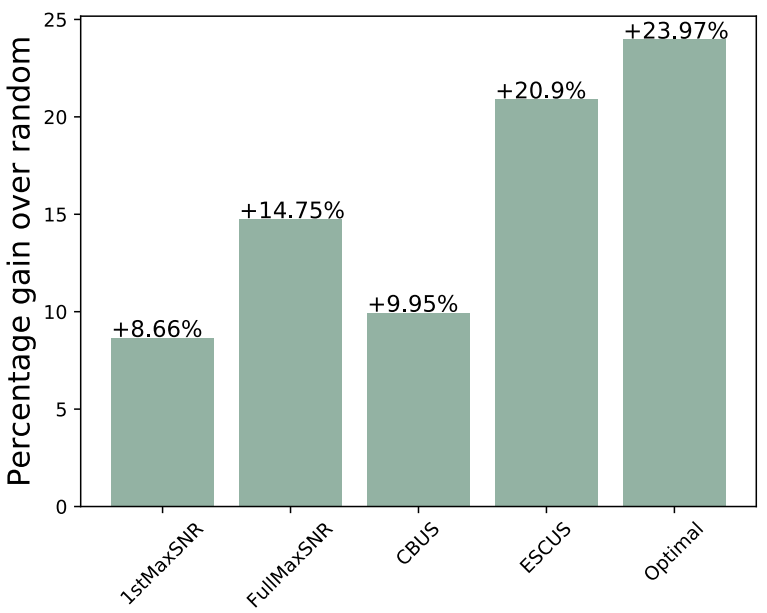

(a) 2 UEs per group

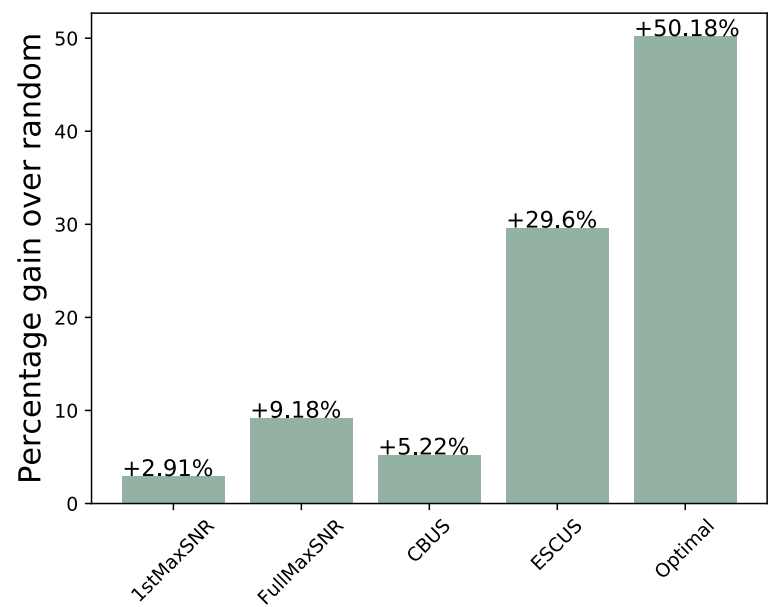

(b) 3 UEs per group

Fig. 2: Algorithms impact on total system capacity

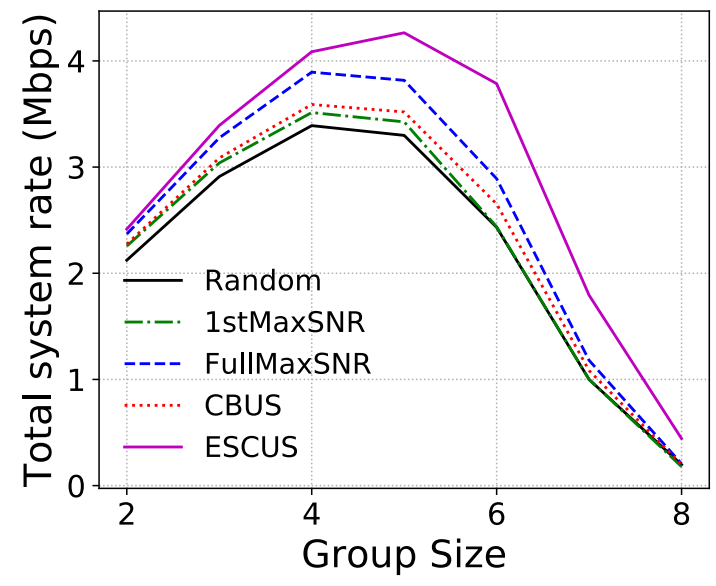

(a) Antenna configuration $n_{r}=4$ and $n_{t}=32$

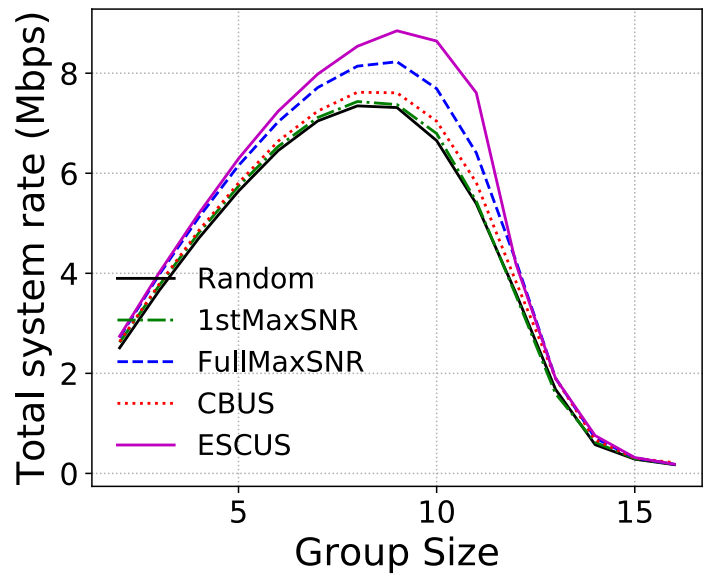

(b) Antenna configuration $n_{r}=4$ and $n_{t}=64$

Fig. 3: Group sizes impact on the system rate (for a $2 \mathrm{MHz}$ bandwidth)

B. Performance evaluation with small number of users per group

Figure 2 shows the percentage gain over a full random solution for different algorithms on the total system rate, using the same parameters and user deployments. With two users per group (see Figure 2a) the gain for all the solutions is significant over a random selection. MaxSNR increases the capacity by $8.7 \%$ when used only on the first user and $14.8 \%$ when used on both users. The use of the correlation on the second user (CBUS) only increases the gain by $1.3 \%$ compared to a random second user. Our ESCUS solution based on previous allocations selection, represents an important gain over all the other solutions, $6.2 \%$ over full MaxSNR, and is only $3.1 \%$ below the optimal strategy. With three users per group (see Figure $2 b$ ) the gain compared to random allocation is lower for all the classical solutions. MaxSNR applied only on the first user is only $2.9 \%$ higher than random allocation and $9.2 \%$, when applied to all users. The solution using the correlation, CBUS, benefits from the high interference environment and is $2.3 \%$ higher than a random selection for secondary users. However, its performance is still $4 \%$ below full system MaxSNR allocation. Our solution is the only one with an increase in its performance, with $20.4 \%$ higher than full MaxSNR, but is $20.6 \%$ below the optimal strategy.

When the number of users per group increases, the level of interference becomes harder to manage. ESCUS maintains its performance when we increase the group size thanks to the knowledge of previous allocations, whereas other solutions see their performance decrease due to their lack of consideration 
for group quality. Finally, the gap between our solution and the optimal selection for three users per group is still large and further work is needed to achieve optimal performance.

\section{Performance evaluation with large number of users per group}

In this section, results with large user group sizes are studied. Users are chosen among a set of 60 users randomly distributed in the cell, thus increasing group diversity compared to the previous setup.

Figure 3 shows the evolution of the total system rate depending on the group size for two antenna configurations. All algorithms show a slow increase up to their best system rate and a fast decrease. Communicating simultaneously with an increasing number of users increases the overall data rate, but also causes interference between users in the same group. When the number of users reaches a given size, interference has a greater impact than the gain from MU-MIMO, leading to a decrease in the total system rate. Depending on the user selection algorithm, this achievable size may differ.

Algorithm performance matches those obtained in section IV-B: random allocation is the worst solution, followed by 1stMaxSNR and then CBUS [3] and finally by FullMaxSNR and our ESCUS solution.

As shown in Figure 3a, the system experiences a strong gain by using ESCUS over FullMaxSNR with an increase of $11.7 \%$, with a group size of 5 users, and an increase of $30.9 \%$, with a group size of 6 users. In Figure 3b, the gain over FullMaxSNR is slightly less with a gain of $7.5 \%$, with a group size of 9 users and $12.4 \%$, with a group size of 10 users.

Compared to correlation based algorithms, ESCUS performs better for all group configurations with a higher total system rate. The results in Figure 3 a show an increase of $18.8 \%$ for ESCUS compared to CBUS regarding the highest value of the total system rate for any group size with $n_{r}=4$ and $n_{t}=32$. Figure $3 \mathrm{~b}$, with $n_{r}=4$ and $n_{t}=64$, shows a lower increase, of $13.4 \%$.

\section{CONCLUSION}

New techniques such as Multi-User-MIMO greatly improve performance over Single-User techniques. To enable the basestation to smartly select users, user selection algorithms are often based on users' channel matrix correlations. In our previous work, we showed that using correlation as an indicator was not suitable in our context. In this paper, we propose a solution called ESCUS (Efficient System Capacity User Selection) based on a new principle which uses previous group allocations as an indicator. This solution is easier to calculate than correlation-based strategies when the number of users per group increases. In terms of performance, ESCUS presents an important improvement compared to classical solutions. A significant part of $5 \mathrm{G}$ depends on services-oriented networks. In future work, we will focus on the integration of Quality of Services metrics in the user selection process to compare our solution to fairness-oriented strategies. Another perspective is to consider channels with estimation errors, where our solution should outperform adverse strategies based on full knowledge of the channel.

\section{REFERENCES}

[1] C.-X. Wang, F. Haider, X. Gao, X.-H. You, Y. Yang, D. Yuan, H. Aggoune, H. Haas, S. Fletcher, and E. Hepsaydir, "Cellular architecture and key technologies for $5 \mathrm{~g}$ wireless communication networks," IEEE Communications Magazine, vol. 52, pp. 122-130, Feb. 2014.

[2] E. Telatar, "Capacity of Multi-antenna Gaussian Channels: Capacity of Multi-antenna Gaussian Channels," European Transactions on Telecommunications, vol. 10, pp. 585-595, Nov. 1999.

[3] T. Ji, C. Zhou, S. Zhou, and Y. Yao, "Low Complex User Selection Strategies for Multi-User MIMO Downlink Scenario," in 2007 IEEE Wireless Communications and Networking Conference, (Kowloon, China), pp. 1532-1537, IEEE, 2007.

[4] Z. Li, P. Li, and K. G. Shin, "MU-MIMO downlink scheduling based on users' correlation and fairness," in 2014 IEEE 25th Annual International Symposium on Personal, Indoor, and Mobile Radio Communication (PIMRC), (Washington DC, USA), pp. 407-412, IEEE, Sept. 2014.

[5] M. Manini, C. Gueguen, R. Legouable, and X. Lagrange, "Study of MIMO channel matrices correlation to optimize resource allocation algorithms in multi-users 5g.," in 2019 12th IFIP Wireless and Mobile Networking Conference (WMNC), IEEE, Sept. 2019.

[6] M. Roy, S. Paquelet, L. Le Magoarou, and M. Crussière, "MIMO channel hardening for ray-based models," in 2018 14th International Conference on Wireless and Mobile Computing, Networking and Communications (WiMob), pp. 1-7, IEEE, 2018.

[7] A. Alkhateeb and R. W. Heath, "Frequency Selective Hybrid Precoding for Limited Feedback Millimeter Wave Systems," IEEE Transactions on Communications, vol. 64, pp. 1801-1818, May 2016.

[8] F. Boccardi and H. Huang, "A Near-Optimum Technique using Linear Precoding for the MIMO Broadcast Channel," in 2007 IEEE International Conference on Acoustics, Speech and Signal Processing - ICASSP '07, (Honolulu, HI), pp. III-17-III-20, IEEE, Apr. 2007.

[9] M. H. A. Khan, K. M. Cho, M. H. Lee, and J.-G. Chung, "A simple block diagonal precoding for multi-user MIMO broadcast channels," EURASIP Journal on Wireless Communications and Networking, vol. 2014, no. 1, p. $95,2014$.

[10] A. M. A. Abdo, X. Zhao, R. Zhang, Z. Zhou, J. Zhang, Y. Zhang, and I. Memon, "MU-MIMO Downlink Capacity Analysis and Optimum Code Weight Vector Design for 5g Big Data Massive Antenna Millimeter Wave Communication," Wireless Communications and Mobile Computing, vol. 2018, pp. 1-12, 2018.

[11] C. Y. Wong, R. S. Cheng, K. B. Lataief, and R. D. Murch, "Multiuser ofdm with adaptive subcarrier, bit, and power allocation," IEEE Journal on selected areas in communications, vol. 17, no. 10, pp. 1747-1758, 1999.

[12] N. Czink, B. Bandemer, G. Vazquez-Vilar, L. Jalloul, C. Oestges, and A. Paulraj, "Spatial separation of multi-user MIMO channels," in Personal, Indoor and Mobile Radio Communications, 2009 IEEE 20th International Symposium on, pp. 1059-1063, IEEE, 2009.

[13] Y. Shimbo, N. Hiruma, H. Suganuma, and F. Maehara, "Control Overhead Reduction Method Employing Frequency Correlation for MU-MIMO-OFDM THP with User Scheduling," in 2018 IEEE 87th Vehicular Technology Conference (VTC Spring), (Porto), pp. 1-5, IEEE, June 2018.

[14] P. Viswanath, D. N. C. Tse, and R. Laroia, "Opportunistic beamforming using dumb antennas," IEEE Transactions on Information Theory, vol. 48, pp. 1277 - 1294, June 2002.

[15] H. Kim, K. Kim, Y. Han, and J. Lee, "An efficient scheduling algorithm for QoS in wireless packet data transmission," in Proc. IEEE Int. Symposium on Personal, Indoor and Mobile Radio Communications (PIMRC), vol. 5, pp. 2244 - 2248, Sept. 2002.

[16] C. Gustafson, K. Haneda, S. Wyne, and F. Tufvesson, "On mm-Wave Multipath Clustering and Channel Modeling," IEEE Transactions on Antennas and Propagation, vol. 62, pp. 1445-1455, Mar. 2014.

[17] 3GPP, "Study on New Radio (NR) access technology," Technical report (TR) 38.912, 3rd Generation Partnership Project (3GPP), 072018. Version 15.0.0. 